\title{
Upregulation of Serum Vascular Endothelial Growth Factor in Patients with Salivary Gland Tumor
}

\author{
Azadeh Andisheh Tadbir, ${ }^{1}$ Bijan Khademi, ${ }^{2}$ Mahyar Malekzadeh, ${ }^{3}$ \\ Maryam Mardani, ${ }^{4}$ and Bahar Khademi ${ }^{5}$ \\ ${ }^{1}$ Department of Oral and Maxillofacial Pathology, School of Dentistry, Shiraz University of Medical Sciences, Ghom Abad, \\ Ghasrodasht Avenue, Shiraz 71956-15878, Iran \\ ${ }^{2}$ Department of Otolaryngology, Khalili Hospital, Shiraz Institute for Cancer Research, Shiraz University of Medical Sciences, \\ Shiraz 71348-45794, Iran \\ ${ }^{3}$ Shiraz Institute for Cancer Research, Shiraz University of Medical Sciences, Shiraz 71348-45794, Iran \\ ${ }^{4}$ Department of Oral Medicine, School of Dentistry, Shiraz University of Medical Sciences, Ghom Abad, Ghasrodasht Avenue, \\ Shiraz 71956-15878, Iran \\ ${ }^{5}$ School of Dentistry, Shiraz University of Medical Sciences (International Branch), Azadi Square, Jahad Sazandegi Street, \\ Shiraz 71447-16699, Iran
}

Correspondence should be addressed to Azadeh Andisheh Tadbir; andisheh@sums.ac.ir

Received 29 May 2013; Accepted 7 July 2013

Academic Editor: Piero Tosi

Copyright (c) 2013 Azadeh Andisheh Tadbir et al. This is an open access article distributed under the Creative Commons Attribution License, which permits unrestricted use, distribution, and reproduction in any medium, provided the original work is properly cited.

Neoangiogenesis is essential for tumor development, invasion, and dissemination. The most potent of the cytokines associated with angiogenesis is vascular endothelial growth factor (VEGF). The aim of the present study was to determine VEGF serum level in patients with salivary gland tumor. Using an ELISA kit, the circulating levels of VEGF in sera from 58 patients with salivary gland tumor and 30 healthy controls were assessed. Mean VEGF levels in sera of patients with salivary gland tumors (574.9 \pm 414.3) were significantly higher than those in controls $(263.9 \pm 310.0)(P=0.009)$. Within the salivary gland tumor group, mean serum VEGF concentration in malignant tumors $(n=27)$ was $727.3 \pm 441.8 \mathrm{pg} / \mathrm{mL}$, and that in benign tumors $(n=31)$ was $442.2 \pm$ $343.3 \mathrm{pg} / \mathrm{mL}$. Mean serum VEGF concentration was significantly higher in malignant tumors than in benign tumors $(P=0.008)$ and was higher in benign tumors than in controls $(P=0.03)$. The data in the present study clearly show that VEGF level was consistently upregulated in benign and malignant tumors in comparison to healthy controls. However, the role of VEGF as a prognostic factor in salivary gland tumor and its application in antiangiogenic therapy require further clinical research.

\section{Introduction}

Neoangiogenesis, the development of new vessels from preexisting ones, is necessary for tumor development, invasion, and dissemination. Angiogenic process not only delivers nutrients for tumor growth but also increases the chance for tumor cells to enter the circulation and metastasis [1].

A variety of signals such as diminished nutrition or oxygen in tissue or genetic changes in oncogenes or tumor suppressor genes can start angiogenic process. Different factors produced from stromal and tumor cells such as growth factors, cytokines, enzymes, and adhesion molecules are involved in this process, and the most potent of them is vascular endothelial growth factor (VEGF) [2].

Among the VEGF family, which consists of VEGF-A, VEGF-B, VEGF-C, VEGF-D, and VEGF-F, VEGF-A is a key angiogenic factor and is mostly used to promote angiogenic phenotype by a tumor [3].

VEGF-A is a dimeric glycoprotein with a molecular weight of $34-45 \mathrm{KDa}$, consisting of two subunits. Increased VEGF expression was seen in different physiologic and pathologic conditions which was associated with hypoxia [4].

VEGF is expressed in several malignant tumors which indicates its importance in angiogenesis process. 
TABLE 1: Serum VEGF level in benign, malignant salivary gland tumors and normal controls as measured by Sandwich ELISA.

\begin{tabular}{lcc}
\hline Groups & Mean serum VEGF $(\mathrm{pg} / \mathrm{mL})$ & $P$ value \\
\hline Controls & $263.9 \pm 310 \pm 0.0$ & Controls versus benign tumors $P=0.03$ \\
Benign salivary gland tumor & $442.2 \pm 343.3$ & Controls versus malignant tumors $P=0.001$ \\
Malignant salivary gland tumor & $727.3 \pm 441.8$ & Benign tumors versus malignant tumors $P=0.008$ \\
\hline
\end{tabular}

Overexpression of VEGF was found in body fluids and solid tumors such as brain, colon, breast, kidney, bladder, lung, and oral cancer [5].

However, no investigations have been conducted regarding serum VEGF levels in patients with salivary gland tumor, so the aim of the present study was to determine VEGF serum level in patients with salivary gland tumor.

\section{Materials \& Methods}

2.1. Subjects. For the purpose of this case-control study, 58 serum samples from the patients diagnosed with salivary gland tumor (24 males, 34 females, age: $44.8 \pm 16.6$ years) consisting of 31 pleomorphic adenoma, 17 adenoid cystic carcinoma, and 10 mucoepidermoid carcinoma and 30 serum samples from healthy control subjects (12 males, 18 females, age: $44.6 \pm 16.6$ years) were collected.

Study subjects were the patients admitted to the Department of Otolaryngology, Khalili Hospital, Shiraz University of Medical Sciences, and diagnosed with salivary gland tumor histopathologically.

Control cases were healthy blood donors, who were matched for age and gender. Exclusion criteria for both groups were based on the presence of any systemic disease, use of corticosteroid or nonsteroid anti-inflammatory medication, or a history of malignancy of any type.

All the participants were informed about the research study and agreed to participate by signing an informed consent form.

2.2. Serum Analysis. Serum samples were obtained from clotted blood following the centrifugation at $4^{\circ} \mathrm{C}$ and storing at $-80^{\circ} \mathrm{C}$ until analysis. VEGF concentrations were measured by Sandwich ELISA, in accordance with the manufacturer's instructions (BMS, GmbH, Germany).

2.3. Statistical Analysis. Statistical analysis was performed using Mann-Whitney test. A $P$ value less than 0.05 was considered significant.

\section{Results}

Mean serum VEGF levels in malignant tumors, benign tumors, and controls are shown in Table 1. Mean VEGF levels in sera of patients with salivary gland tumors $(574.9 \pm 414.3)$ were significantly higher than those in controls (263.9 \pm 310.0) $(P=0.009)$. Within the salivary gland tumor group, mean serum VEGF concentration in malignant tumors $(n=$ 27) was $727.3 \pm 441.8 \mathrm{pg} / \mathrm{mL}$, and that in benign tumors $(n=31)$ was $442.2 \pm 343.3 \mathrm{pg} / \mathrm{mL}$. Mean serum VEGF concentration was significantly higher in malignant tumors than in benign tumors $(P=0.008)$ and was higher in benign tumors than in controls $(P=0.03)$.

No relation was found between mean VEGF levels and sex and age of the patients and controls.

\section{Discussion}

Angiogenesis is necessary for tumor growth. It is a multifaceted process which is regulated by a variety of inducers and inhibitors, which are produced by different cell types like stromal cells, endothelial cells, and tumor cells [6]. Different angiogenic factors have been described. VEGF plays an important role in both pathologic and physiologic conditions [7].

VEGF is one of the most important angiogenic factors involved in tumor angiogenesis. It has been suggested that VEGF is a potent mitogen for endothelial cells and can directly induce new blood vessels formation. Concurrently, increased VEGF will enhance microvascular permeability and will lead to plasma protein extravasation (e.g., fibrinogen) into the extravascular area. Then, extra vascular fibrinogen causes clot formation and other proteins like fibronectin are added to fibrin clot and formed a suitable matrix for growth of endothelial cells [8].

Numerous studies have shown that VEGF is involved in tumor progression. VEGF can increase tumor cell extravasation and metastasis through inducing angiogenesis, enhancing vascular permeability, and stimulating immunosuppression [8-10].

Clearly, identification of different biologic markers and understanding of underlying mechanisms may provide useful markers for determining prognosis and effective treatment.

Recently, different functions such as lymphangiogenesis, enhanced vascular permeability, and antiapoptosis activity have been recognized for VEGF which all have a role in tumor progression $[10,11]$.

VEGF overexpression has been reported in different tumors. Kulapaditharom et al. (2012) found that the most aggressive head and neck squamous cell carcinoma showed the highest VEGF expression. These findings suggest that VEGF has a role in the progression of head and neck squamous cell carcinoma toward an aggressive and invasive phenotype [12].

Lequerica-Fernández et al. (2007) showed that VEGF can contribute to the progression of salivary gland carcinomas and seems to be associated with lymph node metastasis and worse survival [13].

Recently, Ke-Xiong et al. (2011) suggested that inducible nitric oxide synthase can motivate the expression of VEGF, and its expression status may help evaluate tumor malignancy and prognosis of the patient [14]. 
A study conducted by Hao et al. (2010) showed that overexpression of VEGF was seen in adenoid cystic carcinoma and its expression was higher in solid form and in advancedstage group. Also VEGF expression was related to invasion and metastasis and proposed as a prognostic factor [15].

Swelam et al. (2005) suggest that tumor cells of pleomorphic adenoma secrete functional VEGF in different forms that induce their proliferation and differentiation, and VEGF expression is regulated by hypoxic conditions [16].

Recently, circulating VEGF levels have been reported to be a reliable and noninvasive marker of angiogenic activity and were shown to be independent prognostic factors which are correlated with survival in various malignant neoplasms [17], but fewer studies were carried out to evaluate its circulating level.

Shang et al. showed serum VEGF levels to be significantly elevated in oral cancers compared to control group [18].

Dirix et al. (1996) showed that high serum VEGF was associated with the large size of the primary tumor in advanced colorectal cancer [19].

Khademi et al. (2013) reported that increased preoperative or postoperative serum levels of VEGF can predict disease recurrence and are associated with poor disease-free and overall survival of patients with HNSCC [20].

In the present study, ELISA kit was used to measure serum VEGF concentrations in patients with salivary gland tumor. By reviewing the articles, this study was the first report to do so.

Results of this study indicate a significant increase in VEGF level from healthy controls to benign and malignant tumors.

So VEGF levels can significantly discriminate patients from controls and malignant from benign tumors. Also our results suggest that VEGF levels play a role in the progression of salivary gland tumors which was similar to those of Doi et al. (1999) and de Faria et al. (2011) [21, 22].

\section{Conclusion}

The data in the present study clearly show that VEGF level was consistently upregulated in benign and malignant tumors in comparison to healthy controls. However, the role of VEGF as a prognostic factor in salivary gland tumor and its application in antiangiogenic therapy require further clinical research.

\section{Acknowledgments}

This paper has been extracted from Mrs. Bahar Khademi's DDS thesis which was conducted under supervision of Dr. Azadeh Andisheh Tadbir and Dr. Bijan Khademi. This study was approved, registered with ID Grant no. 8591019, and supported by the Vice-Chancellery of International Branch of Shiraz University of Medical Sciences.

\section{References}

[1] S. Pourshahidi, A. A. Tadbir, H. Ebrahimi et al., "Serum level of vascular endothelial growth factor-c in patients with head and neck squamous cell carcinoma," Australian Journal of Basic and Applied Sciences, vol. 5, no. 9, pp. 426-429, 2011.

[2] D. Hodorowicz-Zaniewska, W. Kibil, A. Małek, J. Szpor, J. Kulig, and K. Sztefko, "Evaluation of serum concentrations of vascular endothelial growth factor (VEGF) in breast cancer patients," Polish Journal of Pathology, vol. 63, no. 4, pp. 255-260, 2012.

[3] S. Nayak, M. M. Goel, S. Chandra et al., "VEGF-A immunohistochemical and mRNA expression in tissues and its serum levels in potentially malignant oral lesions and oral squamous cell carcinomas," Oral Oncology, vol. 48, no. 3, pp. 233-239, 2012.

[4] M. Farzin, M. Mardani, J. Ghabanchi et al., "Serum level of matrix metalloproteinase-3 in patients with oral Lichen planus," Iranian Red Crescent Medical Journal, vol. 14, no. 1, 2012.

[5] C.-J. Liu, K.-W. Chang, S.-C. Lin, and H.-W. Cheng, "Presurgical serum levels of matrix metalloproteinase-9 and vascular endothelial growth factor in oral squamous cell carcinoma," Oral Oncology, vol. 45, no. 10, pp. 920-925, 2009.

[6] D. Hanahan and J. Folkman, "Patterns and emerging mechanisms of the angiogenic switch during tumorigenesis," Cell, vol. 86, no. 3, pp. 353-364, 1996.

[7] M. Sawatsubashi, T. Yamada, N. Fukushima, H. Mizokami, O. Tokunaga, and T. Shin, "Association of vascular endothelial growth factor and mast cells with angiogenesis in laryngeal squamous cell carcinoma," Virchows Archiv, vol. 436, no. 3, pp. 243-248, 2000.

[8] Z.-J. Shang, J.-R. Li, and Z.-B. Li, "Upregulation of serum and tissue vascular endothelial growth factor correlates with angiogenesis and prognosis of oral squamous cell carcinoma," Journal of Oral and Maxillofacial Surgery, vol. 65, no. 1, pp. 1721, 2007.

[9] M. E. Baldwin, S. A. Stacker, and M. G. Achen, "Molecular control of lymphangiogenesis," BioEssays, vol. 24, no. 11, pp. 1030-1040, 2002.

[10] J. A. Nagy, E. Vasile, D. Feng et al., "Vascular permeability factor/vascular endothelial growth factor induces lymphangiogenesis as well as angiogenesis," Journal of Experimental Medicine, vol. 196, no. 11, pp. 1497-1506, 2002.

[11] J. E. Ohm, D. I. Gabrilovich, G. D. Sempowski et al., "VEGF inhibits T-cell development and may contribute to tumorinduced immune suppression," Blood, vol. 101, no. 12, pp. 48784886, 2003.

[12] B. Kulapaditharom, V. Boonkitticharoen, and C. Sritara, "Plasma vascular endothelial growth factor dysregulation in defining aggressiveness of head and neck squamous cell carcinoma," Journal of Oncology, vol. 2012, Article ID 687934, 7 pages, 2012.

[13] P. Lequerica-Fernández, A. Astudillo, and J. C. De Vicente, "Expression of vascular endothelial growth factor in salivary gland carcinomas correlates with lymph node metastasis," Anticancer Research, vol. 27, no. 5 B, pp. 3661-3666, 2007.

[14] O. Y. Ke-Xiong, L. Jun, and H. Zhi-Quan, "Association of clinicopathologic parameters with the expression of inducible nitric oxide synthase and vascular endothelial growth factor in mucoepidermoid carcinoma," Oral Diseases, vol. 17, no. 6, pp. 590-596, 2011.

[15] L. Hao, N. Xiao-lin, C. Qi, Y. Yi-ping, L. Jia-quan, and L. Yanning, "Nerve growth factor and vascular endothelial growth factor: retrospective analysis of 63 patients with salivary adenoid cystic carcinoma," International journal of oral science, vol. 2, no. 1, pp. 35-44, 2010.

[16] W. Swelam, H. Ida-Yonemochi, S. Maruyama, K. Ohshiro, J. Cheng, and T. Saku, "Vascular endothelial growth factor in 
salivary pleomorphic adenomas: one of the reasons for their poorly vascularized stroma," Virchows Archiv, vol. 446, no. 6, pp. 653-662, 2005.

[17] R. T.-P. Poon, S.-T. Fan, and J. Wong, "Clinical implications of circulating angiogenic factors in cancer patients," Journal of Clinical Oncology, vol. 19, no. 4, pp. 1207-1225, 2001.

[18] Z.-J. Shang, J.-R. Li, and Z.-B. Li, "Circulating levels of vascular endothelial growth factor in patients with oral squamous cell carcinoma," International Journal of Oral and Maxillofacial Surgery, vol. 31, no. 5, pp. 495-498, 2002.

[19] L. Y. Dirix, P. B. Vermeulen, G. Hubens et al., "Serum basic fibroblst growth factor and vascular endothelial growth factor and tumour growth kinetics in advanced colorectal cancer," Annals of Oncology, vol. 7, no. 8, pp. 843-848, 1996.

[20] B. Khademi, M. Soleimanpour, A. Ghaderi, and M. Mohammadianpanah, "Prognostic and predictive value of serum vascular endothelial growth factor (VEGF) in squamous cell carcinoma of the head and neck," Oral and Maxillofacial Surgery, 2013.

[21] R. Doi, I. Kuratate, E. Okamoto, K. Ryoke, and H. Ito, "Expression of p53 oncoprotein increases intratumoral microvessel formation in human salivary gland carcinomas," Journal of Oral Pathology and Medicine, vol. 28, no. 6, pp. 259-263, 1999.

[22] P. R. de Faria, R. A. Lima, F. L. Dias et al., "Vascular endothelial growth factor and thymidine phosphorylase expression in salivary gland tumors with distinct metastatic behavior," Journal of Oral Pathology and Medicine, vol. 40, no. 6, pp. 456-459, 2011. 


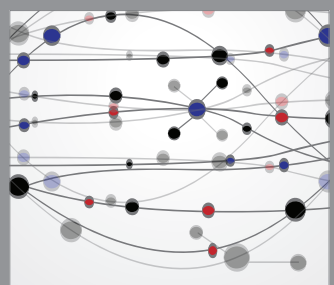

The Scientific World Journal
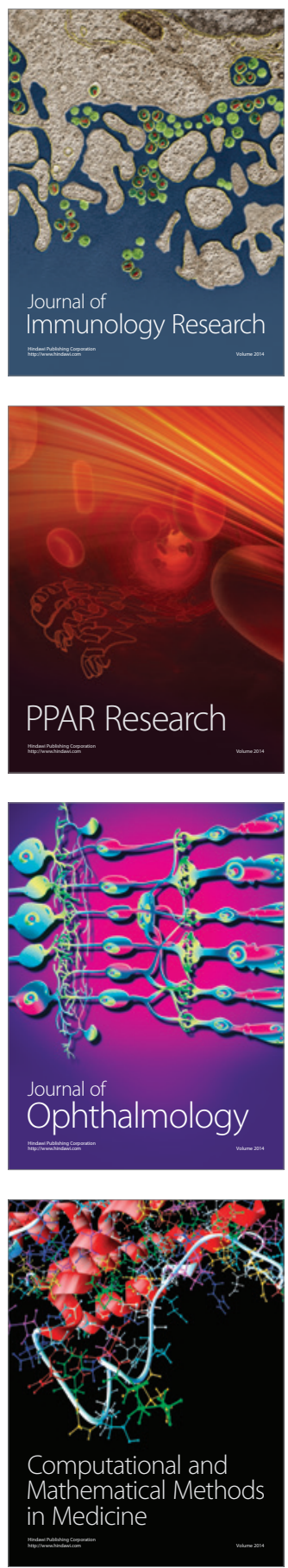

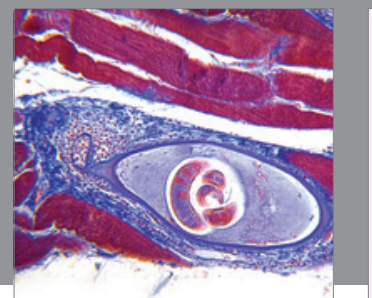

Gastroenterology

Research and Practice
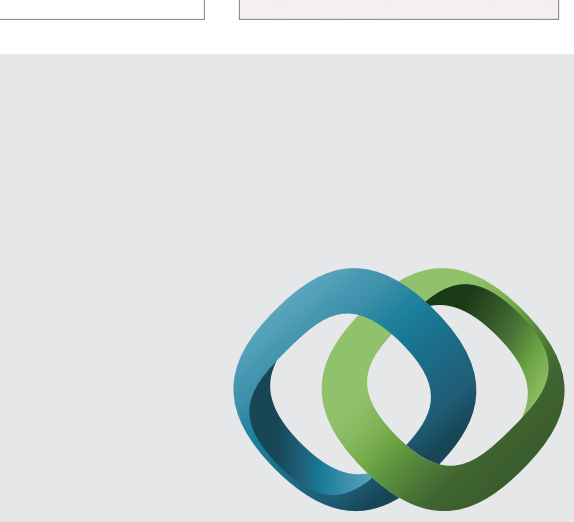

\section{Hindawi}

Submit your manuscripts at

http://www.hindawi.com
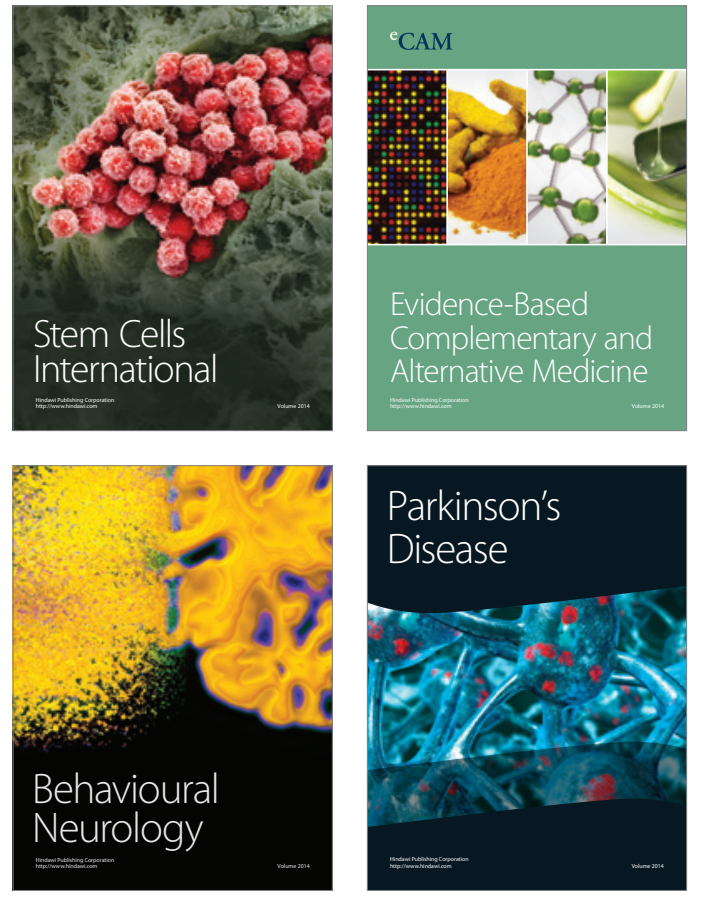
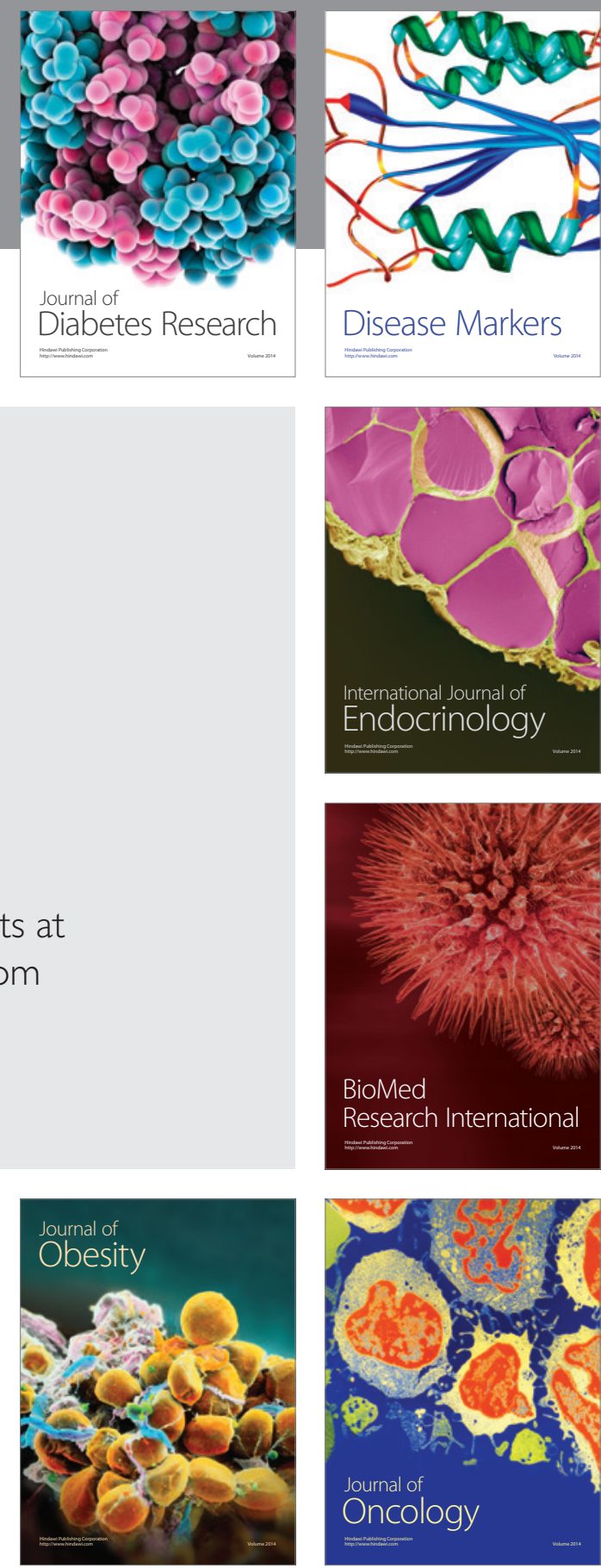

Disease Markers
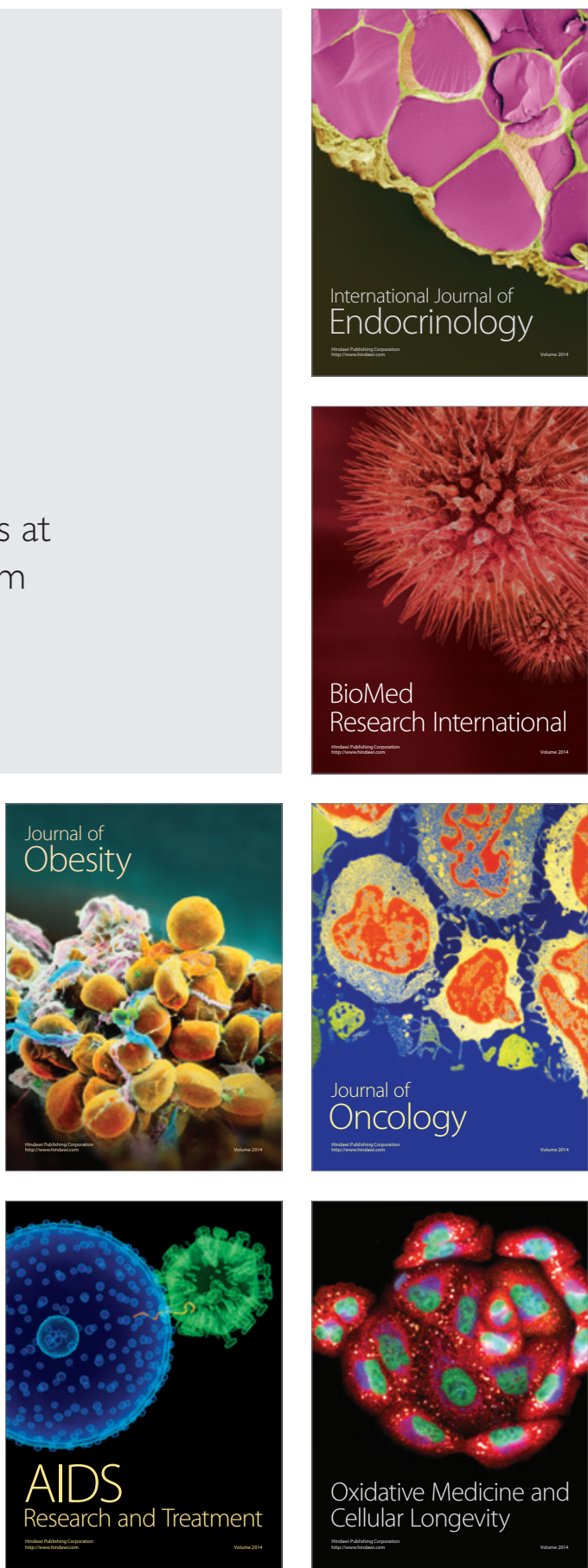